\title{
Top-Off Injection and Higher Currents at the Stanford Synchrotron Radiation Lightsource
}

\author{
Johannes M. Bauer, James C. Liu, Alyssa A. Prinz, Sayed H. Rokni \\ SLAC National Accelerator Laboratory, Radiation Protection Department, \\ MS 48, 2575 Sand Hill Road, Menlo Park, CA 94025, U.S.A. \\ bauerj@slac.stanford.edu
}

\section{INTRODUCTION}

The Stanford Synchrotron Radiation Lightsource (SSRL) at the SLAC National Accelerator Laboratory is a $234 \mathrm{~m}$ circumference storage ring for $3 \mathrm{GeV}$ electrons with its synchrotron radiation serving currently 13 beamlines with about 27 experimental stations. It operated for long time with $100 \mathrm{~mA}$ peak current provided by usually three injections per day. In July 2009, the maximum beam current was raised to $200 \mathrm{~mA}$. Over the period from June 2009 to March 2010, Top-Off operation started at every beamline. Top-Off, i.e., the injection of electrons into the storage ring with injection stoppers open, is necessary for SSRL to reach its design current of $500 \mathrm{~mA}$. In the future, the maximal power of the injection current will also soon be raised from currently $1.5 \mathrm{~W}$ to $5 \mathrm{~W}$.

The Radiation Protection Department at SLAC worked with SSRL on the specifications for the safety systems for operation with Top-Off injection [1] and higher beam currents.

\section{TOP-OFF OPERATION}

Whenever beamline injection stoppers are closed at a synchrotron storage ring like at SSRL, the photon beam is intercepted, and the optics of the beamline may change its alignment due to change in thermal flux. While this alignment change is negligible at low beam currents (for example at $100 \mathrm{~mA}$ ), it becomes disruptive at higher currents like $500 \mathrm{~mA}$. The current infrequent injection pattern (as shown at the bottom of Figure 1) becomes untenable at higher currents. Frequent injection with injection stoppers open is therefore essential to improving SSRL's performance.

\section{Ray Trace Analysis}

The possibility for injected electrons to enter the beamlines and reach the user floor while beam is stored in the ring is considered to be remote, but could not be fully excluded. A ray trace analysis was therefore developed by SSRL in collaboration with Lawrence Berkeley National Laboratory and applied for each beamline to determine which magnet settings allow beam to reach which point for the given geometry. Based on this, a "Beam Containment System" (BCS) was developed to

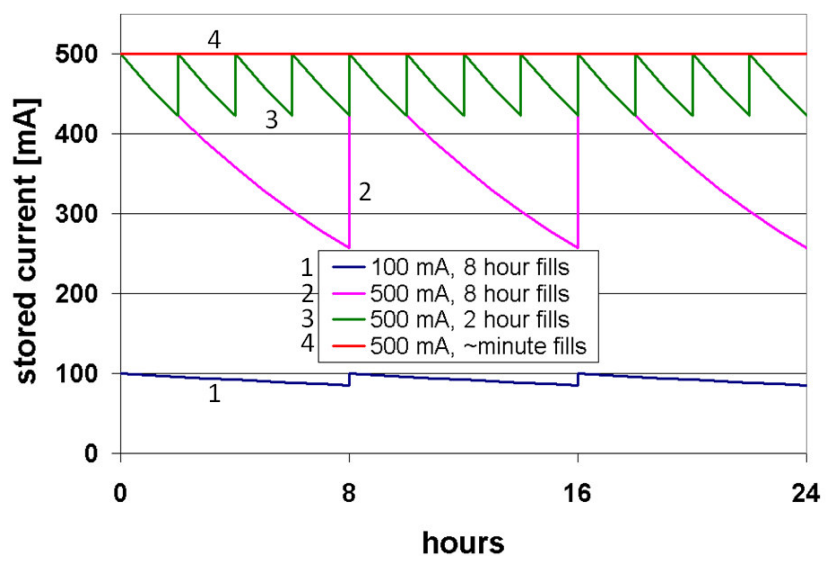

Figure 1: Schematics drawing of injection patterns for current $100 \mathrm{~mA}$ (1) and future $500 \mathrm{~mA}$ operation (2-4).

enforce conditions that ensure injected beam would not pass beyond certain "Safety Endpoints" within the storage ring.

\section{Radiological Considerations}

Three situations had to be considered for radiation protection:

- Radiation from normal operation due to Bremsstrahlung created by injected beam inside the storage ring: This radiation was measured during strictly controlled Top-Off tests and extrapolated to future $500 \mathrm{~mA}$ top-off operation, taking into account that the current lost (and current to be replenished) increases more than linearly because of decreasing lifetimes with higher currents. In the end, the dose from all sources should not exceed $1 \mathrm{mSv}$ (100 mrem) in 1000 hours on the user floor.

- Radiation from beam that is mis-steered, but is still within the envelope defined by the BCS, and that does not pass the Safety Endpoint: Monte Carlo simulations (like the one shown in Figure 2) determined this dose to be maximal a few rem (few tens of $\mathrm{mSv}$ ) per hour for the future maximal injection beam power of $5 \mathrm{~W}$. Due to the unlikely nature and the presence of an interlocked radiation monitor that shuts off beam on high dose rates, this dose rate level is considered to be acceptable.

Presented at Nevada Section of the American Nuclear Society 2010 Topical Meeting, April 18-23, 2010, Las Vegas, NV, USA Accepted for publication in Nuclear Technology

Work supported in part by US Department of Energy contract DE-AC02-76SF00515. 
- Radiation due to beam loss on the user floor: Electrons can reach the experimental floor only with a complete BCS system failure and simultaneous intentional mis-steering. If both the hardware failure and the intentional mis-steering would ever happen such that electron beam arrives at the user floor, interlocked radiation monitors will limit the duration to less than two seconds. Monte Carlo code simulations estimate for this case dose rates of up to $3.3 \mathrm{~Sv} / \mathrm{h}(330 \mathrm{rem} / \mathrm{h})$ for $5 \mathrm{~W}$ injection beam power. Integrated over two seconds, personnel would then be exposed to maximal $2 \mathrm{mSv}$ (200 mrem), which is considered to be acceptable for this very remote situation.

\section{Top-Off Safety Systems}

The Top-Off BCS consists of several components. At least $50 \mathrm{~mA}$ of electron beam must be stored in the ring before Top-Off may commence. The power supplies of numerous magnets are interlocked to stay within specific voltage and current limits. These interlocked magnets are magnets located in the BTS (booster-toSPEAR) line, that carries the injected beam to SPEAR, and are the main dipole magnets of the ring, individual quadrupole and sextupole magnets, various corrector magnets, and newly-installed clearing magnets at dipole beam lines that bend away any electron that might make it into the beamline.

The above-mentioned radiation monitors are also part of the system. They stop Top-Off injection if they detect a dose rate above $0.02 \mathrm{mSv} / \mathrm{h}(2 \mathrm{mrem} / \mathrm{h})$ outside the photon beamline [2].

An additional Top-Off Safety System (but not part of the BCS) is a software dose interlock that inhibits TopOff injection once a radiation monitor records a total dose of 1 mrem during the day.

\section{Radiation Measurements During Top-Off Injection}

Before Top-Off injection was permitted during user runs, tests were performed under strict radiological controls. The tests found at some beamlines dose rates of

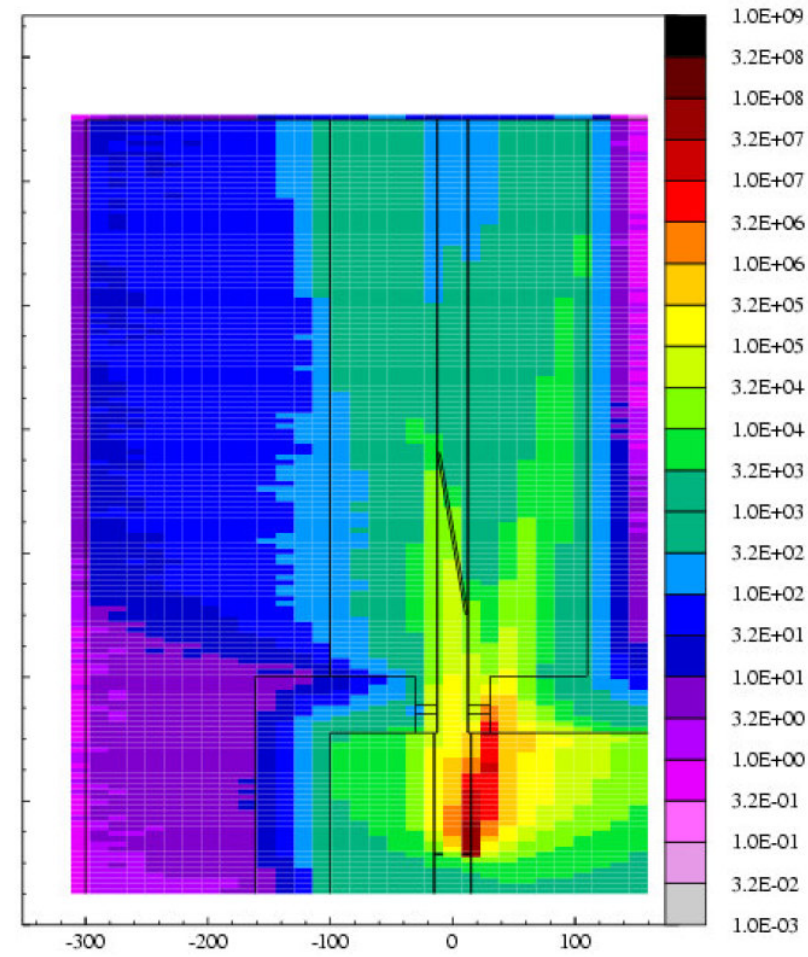

Figure 2: Example of Monte Carlo simulation with $1 \mathrm{~W}$ beam hitting a mask of the beamline structure inside the SPEAR enclosure with 2 -feet thick concrete walls. The beamline hutch outside the SPEAR enclosure is in the upper $3 / 4$ of the plot. During operation, personnel can access the hutch wall (in plot along the vertical line at $100 \mathrm{~cm})$. The dose rates are given in mrem/h $(0.01 \mathrm{~Sv} / \mathrm{h})$. Future injection power may reach $5 \mathrm{~W}$.

up to $18 \mathrm{microSv} / \mathrm{h}(1.8 \mathrm{mrem} / \mathrm{h})$ during good injection, up to $30 \mathrm{microSv} / \mathrm{h}(3 \mathrm{mrem} / \mathrm{h})$ during inefficient injection (Figure 3).

Subsequent improvement by SSRL to the injector system, especially better beam optics and lattice and the removal of vacuum windows, reduced these dose rates to $1.6 \mathrm{microSv} / \mathrm{h}(0.16 \mathrm{mrem} / \mathrm{h})$ maximal for good injection, $4.8 \mathrm{microSv} / \mathrm{h}(0.48 \mathrm{mrem} / \mathrm{h})$ for inefficient injection. This dose rate is low enough for user operation, and TopOff operation commenced in July 2009 on the first

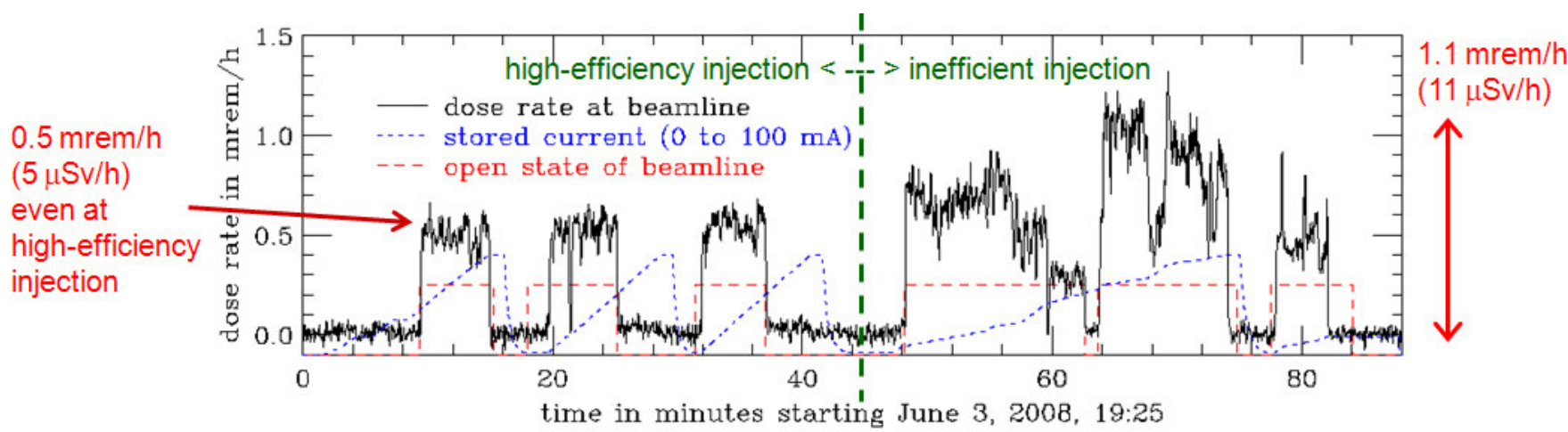

Figure 3: Data from top-off test showing in the readings of radiation monitors outside the beamline (see arrow). The dose rate was seen in this case to rise by $5 \mathrm{microSv} / \mathrm{h}(0.5 \mathrm{mrem} / \mathrm{h})$ during high-efficiency injection (left part of plot), and by $11 \mathrm{microSv} / \mathrm{h}(1.1 \mathrm{mrem} / \mathrm{h})$ during inefficiency injection (right part of plot). 
beamlines. By March 2010, all beamlines had been analyzed for safety, their radiation levels measured and their BCS implemented. Tests started with beam injected no longer every ten minutes, which keeps the SPEAR3 current stable within $1 \%$ at $200 \mathrm{~mA}$.

\section{OPERATION AT HIGHER BEAM CURRENTS}

High beam currents raise the demands on the shielding for the hutches and carry bigger risks for burn-through due to the higher synchrotron radiation flux.

\section{Radiological Considerations}

All hutches have been designed or upgraded to meet the shielding requirements for $500 \mathrm{~mA}$. Measurements were obtained of the radiation created by Gas Bremsstrahlung during normal stored beam operation and passing through the beam pipe onto the floor; extrapolation to future $500 \mathrm{~mA}$ stored current operation indicated that dose rates would exceed the limit of $1 \mathrm{mSv}$ (100 mrem) in 1000 hours on the user floor. Additional shielding is therefore planned to be added for higher currents. Note that this radiation is due to just stored current, not due to top-off operation.

\section{Beamline Beam Containment System}

The higher photon beam power at stronger currents increases the heat load on the components that intercept the beam. During the beamline design, care has been taken to identify through ray traces all devices on which synchrotron radiation beam might terminate. Those devices have to either be shown to be able to withstand the incoming thermal power indefinitely without cooling, or have to be actively cooled. A Beam Containment System with control by a PLC (Programmable Logic Controller), all managed with strict configuration control, has been implemented to monitor those cooling water flows. It closes the injection stoppers if the flow is interrupted for more than three seconds, and initiates dumping of the stored beam if the injection stoppers have not closed after 17 seconds. Similarly, sensors monitor the vacuum pressure on each beamlines. If they detect higher than normal pressure (which might indicate that burn-through of the vacuum enclosure has happened), the system shuts off the stored beam at once unless injection stoppers are already closed.

\section{Beam Tests}

First beam tests have been performed at some beamlines at currents of up to $350 \mathrm{~mA}$. Beamline engineers tested thermal stability and optical properties of the beamline optics, while Radiation Protection personnel carefully measured the radiation outside the beamline shielding. Dose rates were found to be below the design limits of $1 \mu \mathrm{Sv} / \mathrm{h}(0.1 \mathrm{mrem} / \mathrm{h})$ at beamline sides. The beam tests are continuing. Once all beamlines have been shown to be safe at the higher currents, user operation will proceed with this higher current. Operation at $500 \mathrm{~mA}$ is expected by Fall 2010.

\section{OPERATION AT HIGH INJECTION CURRENTS}

While for continuous top-off injection the current limit of $1.5 \mathrm{~W}$ on the injection beam power is sufficient, future fills from 0 to $500 \mathrm{~mA}$ (required, e.g., after unexpected beam loss) will be greatly accelerated by higher injection power. Plans ask for a maximum of $5 \mathrm{~W}$ in injection power. Shielding is already being upgraded, and BCS systems (including a Long Ion Chamber around the SPEAR ring) will be set up to provide for safe operation at $5 \mathrm{~W}$ injection beam power.

\section{CONCLUSION}

Implementation of Top-Off BCS and improvements in the SSRL injector system have permitted Top-Off injection with negligible radiation levels to the users. Top-Off operation is currently taking place for all of SSRL's beamlines and the time between injections has been reduced during tests from 8 hours to 10 minutes, which keeps the stored current constant to $1 \%$.

The maximal beam current during user operation was raised in July 2009 from $100 \mathrm{~mA}$ to $200 \mathrm{~mA}$. A Beamline $\mathrm{BCS}$ is in place that permits operation up to $500 \mathrm{~mA}$. Testing of beamlines, including radiation surveys, is currently taking place for these higher currents.

Preparations are being made to raise the maximum injection beam power from currently $1.5 \mathrm{~W}$ to $5 \mathrm{~W}$.

\section{REFERENCES}

1. J. SAFRANEK et al., "SPEAR3 Accelerator physics update," PAC07-TUPMS055, SLAC-PUB-12949, Proceedings of Particle Accelerator Conference (PAC 07), Albuquerque, New Mexico, U.S.A., June 2007.

2. J.M. BAUER et al., "New Radiation Monitors at SLAC," presentation this conference.

\section{ACKNOWLEDGMENTS}

The authors thank SSRL and the staff working there for the excellent cooperation on this project and congratulate them on their success in improving SSRL's performance. This work is supported by Department of Energy Contract DE-AC02-76SF00515. 